Philosophia Scientie

\section{Philosophia Scientiæ}

Travaux d'histoire et de philosophie des sciences

CS $7 \mid 2007$

Louis Rougier : vie et œuvre d'un philosophe engagé

\title{
Louis Rougier tel que je l'ai connu
}

\section{Béatrice Fink}

\section{(2) OpenEdition}

\section{Journals}

Édition électronique

URL : http://journals.openedition.org/philosophiascientiae/424

DOI : 10.4000/philosophiascientiae.424

ISSN : 1775-4283

Éditeur

Éditions Kimé

Édition imprimée

Date de publication : 1 janvier 2007

Pagination : 4-7

ISBN : 978-2-84174-412-1

ISSN : $1281-2463$

Référence électronique

Béatrice Fink, «Louis Rougier tel que je l'ai connu », Philosophia Scientiæ [En ligne], CS 7 | 2007, mis en ligne le 08 juin 2011, consulté le 15 janvier 2021. URL : http://journals.openedition.org/ philosophiascientiae/424; DOI : https://doi.org/10.4000/philosophiascientiae.424 


\title{
Louis Rougier tel que je l'ai connu
}

\author{
Béatrice Fink \\ Université de Maryland/USA
}

Tout le monde connaît l'histoire des chaussettes d'Einstein. Derrière l'homme d'idées et l'homme d'action se trouve l'homme tout court. Autrement exprimé, il y a l'homme de l'espace public et celui de l'espace privé. C'est vers ce dernier que je me tourne, vers mon beau-père, deuxième époux de ma mère Lucie qui fut, elle, sa troisième épouse. Il ne s'agit ici ni d'un sketch biographique ni d'un constat mais d'un homme vu à travers le prisme de sa belle-fille.

Pour moi il y avait non un mais plusieurs Louis. Tout d'abord, celui de la famille et du foyer. Issu d'un milieu bourgeois de Lyon, il entretint sa vie durant des rapports étroits avec ses cinq frères et sIJurs et leurs familles respectives. ${ }^{1}$ Seul universitaire et philosophe des six enfants, «l'oncle Louis » fut toujours l'objet du respect et de l'admiration de l'entourage familial malgré son infidélité à sa ville natale, puisqu'il opta bien vite pour Paris. Côté foyer, c'est-à-dire New York et Paris en ce qui me concerne, j'ai vite compris, même petite, qu'entre lui et ma mère c'était le grand amour. ${ }^{2}$ Et, chose rare, cela a duré. Il était pour elle

Philosophia Scientice, Cahier spécial 7, 2007, 4-7.

\footnotetext{
${ }^{1}$ Son neveu Jacques Rougier détient une collection de lettres que le jeune Louis écrivit à sa mère qui témoignent des rapports étroits d'affection qu'il avait également avec celle-ci. J'eus souvent l'occasion de rencontrer les membres de la famille Rougier.

${ }^{2}$ Bien que Louis soit entré dans ma vie à la veille de la $2^{\mathrm{e}}$ guerre mondiale - plus
} 
plein d'attentions et d'une tendresse sans relâche. Elle, de son côté, recevait souvent à dîner dans notre appartement New Yorkais de nombreux intellectuels français et viennois s'étant réfugiés en Amérique comme lui durant les années de guerre. ${ }^{3}$ Pour parer aux soucis chroniques d'argent elle prit comme pensionnaires des écoliers du Lycée Français de New York et donna des leçons particulières de français à des Américaines de sa connaissance. Louis, de son côté, maintint tant qu'il le put en ces temps difficiles ses activités d'enseignant et d'écrivain.

L'homme du foyer ne se révéla pourtant pleinement qu'à Paris. Je le voyais plus et, étant plus mûre, me sentais plus apte à discuter avec lui. A la maison, il menait une vie quotidienne d'une grande simplicité. Travaux de lecture et d'écriture dans son bureau, souvent tard dans la nuit. Course du matin pour chercher le Figaro et la baguette; repas frugaux à domicile; promenade du soir pour «s'aérer » et prendre un café. Le tout entrecoupé de visites d'amis et chez des amis. Une gamme très diversifiée de gens défilaient au 354, rue St. Honoré : importants ou non, Français ou étrangers, intellectuels, hommes d'affaires, artistes, certains de mes collègues et étudiants, petites gens du quartier, sans parler des jeunes sous-locataires souvent invités à se joindre aux autres. On s'attardait autour des repas et goûters style viennois que ma mère savait si bien préparer suivis de longues causeries au salon qui tournaient à l'occasion en de véritables débats.

Causeries et débats ne se limitaient pas à ces heures post-repas. Le beau-père que je connaissais devint pour moi durant de longues années un interlocuteur privilégié. Vu son intarissable curiosité d'esprit il tenait à savoir dès mon arrivée sur place les dernières nouvelles tous azimuts. ${ }^{4}$ Nous discutions donc des multiples aspects de la vie américaine, des travaux de mon mari (qui était économiste), de mes propres travaux et échanges intellectuels. Ce fut lui qui m'initia le premier à la pensée politique libérale de Benjamin Constant dont je fis par la suite le sujet de ma thèse de doctorat et de maints articles. ${ }^{5}$ A l'époque - nous sommes au milieu des années soixante - cette pensée était fort peu connue et

particulièrement durant les mois passés en France durant 1938 et 1939 - je n'ai de lui que des souvenirs indistincts de cette époque vu le très jeune âge que j'avais.

${ }^{3}$ Louis épousa me mère à New York en décembre 1942.

${ }^{4}$ Étant restée en Amérique après la guerre, y ayant fondé ma propre famille et fait ma carrière universitaire, je passais presque tous mes étés avec mes parents Rougier, même parfois l'année entière lors d'une bourse d'études ou d'un congé sabbatique.

${ }^{5}$ Cette pensée est évoquée ou citée dans plus d'un écrit de mon beau-père. Il existe d'ailleurs des notes manuscrites de lui qui prêtent à croire qu'il avait une fois en tête le projet de préparer une édition d'ouvrages de Constant pour publication. Tel est du moins ce que pense Claudia Berndt, dont la thèse de doctorat sur la philosophie de Louis Rougier est en bonne voie. 
loin de jouir de la vogue intellectuelle qui la rehausse aujourd'hui. Je notais jusqu'à quel point les opinions de Louis se formaient à base de l'expérience concrète plutôt que de telle ou telle doctrine. Il reconnaissait, par exemple, à propos de la Chine maoiste qu'elle «avait gagné la bataille des mouches ». D'autre part, lorsqu'en jeune enthousiaste il visita l'Union soviétique au début des années 30 et vit que de vieux journaux remplaçaient les chaussures des passants, il comprit ce que la nouvelle idéologie avait de dangereux.

Les deux exemples cités ci-dessus sont en fait tirés de souvenirs et d'anecdotes racontés de manière fort animée. Parmi ceux-ci, il y en a un qui frappe particulièrement. Il s'agit d'une visite qu'il rendit à celui qui occupait toute sa pensée à l'époque, à savoir Henri Poincaré. ${ }^{6}$ Après un entretien qui lui fut gracieusement accordé au domicile de ce dernier un jour où il pleuvait, il partit et avait presque atteint la sortie de l'immeuble lorsque Poincaré, qui essayait de le rattraper parapluie en main, lui cria «Monsieur, vous avez oublié ceci». Un souvenir resté fort vif et démultiplié par le nombre de fois qu'il fut raconté!

La troisième facette du prisme me révèle le globe-trotter, l'amateur des dépaysements, de natures et de cultures autres. Sa zone de prédilection était la Méditerranée, et ma mère disait non sans fierté qu'il y connaissait - pour y avoir vécu ou les avoir visité avec elle — tous les pays riverainš̌ à l'exception de l'Albanie. Que de fois ai-je entendu Louis évoquer «Alger la blanche» ou les promenades du Pincio à Rome. J'eus le bonheur de visiter cette ville avec lui, ainsi que d'autres hauts lieux italiens. La visite était rude car, avec lui, s'il ne restait plus assez de temps pour le repas, eh bien, c'était tant pis. A l'art et à la culture se mêlait toutefois toujours la nature car il avait son côté romantique et rêveur. Des collines de Rome ou celles de Toscane aux sommets alpins il n'y avait d'ailleurs qu'un pas. Nous penchions toujours pour la montagne plutôt que la mer, par atavisme et par amour des longues randonnées. ${ }^{7}$ Le plus souvent c'était l'Autriche pour faire plaisir à ma mère, souvenirs d'enfance oblige. Louis aurait préféré des endroits aux ciels plus bleus et se débrouillait mal en allemand mais s'y pliait avec grâce, et aussi avec une grande générosité : non seulement moi mais plus tard mes enfants étaient toujours ses invités. Dans la foule des souvenirs de ces étés heu-

\footnotetext{
${ }^{6} \mathrm{Je}$ n'ai malheureusement pas retenu la date exacte mais il s'agit vraisemblablement d'un moment peu avant la disparition du grand mathématicien, c'est-à-dire lorsque la thèse que mon beau-père préparait sur lui l'occupait à part entière.

${ }^{7}$ Louis avait escaladé le Mont Blanc au début du $20^{\mathrm{e}}$ siècle, à une époque où les dames alpinistes portaient encore des robes longues! Quant à ma mère et moi, nos racines autrichiennes nous ont attachées aux Alpes. Mon fils cadet est alpiniste, parapentiste et delta-planiste.
} 
reux il y a Louis qui, en toute sérénité, lisait ou écrivait dans le jardin de notre modeste location aux moments de repos. Les premières vacances de l'après-guerre passées avec lui et ma mère - en fait mon premier retour en Europe - furent pourtant d'un ordre différent. Cela se passait en 1950 dans un hôtel situé sur le beau plateau d'Igls au-dessus d'Innsbruck. A cette époque l'Autriche était encore occupée par les Alliés, et le Tyrol se trouvait en zone française. A notre surprise, lors de notre arrivée, on nous annonça que la réservation initiale avait été annulée et que la plus belle chambre, avec balcon et vue inégalée avait été gracieusement mise à la disposition du couple Rougier par le général Béthouard, et ceci pendant un mois entier. Je suis retournée à cet endroit avec ma mère peu avant sa mort. L'hôtel Iglerhof avait été converti en un banal ensemble d'appartements face au parking des résidents.

Je vis Louis pour la dernière fois durant l'été précédant sa disparition en octobre 1982. J'étais de retour en Amérique lorsqu'un coup de téléphone de ma mère m'annonça sa disparition. Elle, qui était à son chevet, me dit que ses derniers mots avaient été ; «c'est fini ». Cela résume à mon avis la clarté d'esprit, le courage et le pragmatisme de mon beau-père philosophe. 